\title{
Nicotine associated breast cancer in smokers is mediated through high level of EZH2 expression which can be reversed by methyltransferase inhibitor DZNepA
}

Kanchan Kumari ${ }^{1,2}$, Biswajit Das ${ }^{3,4}$, Amit Adhya ${ }^{5}$, Sanjib Chaudhary ${ }^{6}$, Shantibhusan Senapati ${ }^{3}$ and Sandip K. Mishra ${ }^{1}$

\begin{abstract}
Recent studies show substantial growth-promoting properties of nicotine (NIC) in cancer, which is a combined outcome of genetic and epigenetic alterations. However, the role of epigenetic modifiers in response to NIC in breast cancer is less studied. In the present study, for the first time we have shown NIC-induced enhanced EZH2 expression. Six pairs of smoking-associated breast cancer patient tissues were analyzed. Samples from smoking breast cancer patients showed distinguished enhanced EZH2 expression in comparison to non-smoking ones. The upregulation in EZH2, which is due to NIC, was further confirmed in breast carcinoma cell lines using $10 \mu \mathrm{M} \mathrm{NIC}, 1 \mu \mathrm{M}$ DZNepA, and EZH2si. The upregulation of EZH2 was concomitant with upregulation in Myc and a9-nAChR. The xenograft of breast cancer cells in BALB/C nude mice in the presence or absence of NIC showed significantly higher tumor uptake in the $\mathrm{NIC}$ injected group, which clearly demonstrates the effect of NIC in breast cancer progression. Interestingly, DZNepA considerably suppressed the NIC-mediated tumor growth. CHIP-qPCR assay confirmed the increased Myc enrichment on EZH2 promoter upon NIC treatment, thereby strengthening our findings that there exists an association between $\mathrm{NIC}, \mathrm{Myc}$, and EZH2. Overall, the present study identifies a strong association between NIC and EZH2 particularly in the progression of breast cancer in smokers through a novel axis involving nAChR and Myc. Moreover, the findings provide preliminary evidence suggesting potential of high level of EZH2 expression as a prognostic marker in smoking-associated breast cancer.
\end{abstract}

\section{Introduction}

Breast cancer is the most common cancer in females. According to the cancer facts and figures provided by the American Cancer Society for the year $2017^{1}$, there are 40,610 and 252,710 number of estimated deaths and new cases respectively for breast cancer in females. It stands at third position in mortality and second for new cases in USA. Regardless of great achievements in the area of

\footnotetext{
Correspondence: Sandip K. Mishra (sandipkmishra@hotmail.com)

${ }^{1}$ Cancer Biology Laboratory, Department of Gene Function and Regulation, Institute of Life Sciences, Bhubaneswar, Odisha, India

${ }^{2}$ Utkal University, Bhubaneswar, Odisha, India

Full list of author information is available at the end of the article Edited by J Chipuk
}

cancer research, there is a big question about the causal of the disease. Exploration of the research findings broadly categorizes the causal factors for breast cancer into genetic and environmental. In-between these two factors there exists another factor called epigenetics that play a significant role in disease progression. Among the list of factors that are preventable, smoking is the one, which is being increasingly prevalent in females round the world including developing countries like India ${ }^{2}$. Smoking is consistently linked to increased breast cancer risk ${ }^{3-5}$. Cigarette smoke consists of more than hundreds of constituents ${ }^{6}$ among which nicotine (NIC) has been widely studied for its effects on neurons ${ }^{7}$. NIC is studied for its unfavorable effects on breast cancer too ${ }^{8-10}$. Association 
of active and passive smoking with breast cancer is reported $^{11,12}$. Nicotinic acetylcholine receptors (nAChR) are the receptors through which NIC functions and are upregulated by it $^{13}$. During NIC-induced transformation of normal breast epithelial cells, overexpression and activation of $\alpha 9$-nAChR is reported ${ }^{14,15}$. Research in this area has shown that NIC makes cancer cells more aggressive $^{16}$.

Covalent modifications of histone proteins play a fundamental role in structure and function of chromatin. One such modification that is central to gene regulation is histone methylation ${ }^{17}$, which is carried out by histone methyltransferases. Polycomb group protein enhancer of zeste homolog 2 (EZH2) is a histone methyltransferase enzyme that tri-methylates histone $\mathrm{H} 3$ at lysine 27 leading to gene repression. Remarkable oncogenic role of EZH2 is reported in several studies ${ }^{18,19}$. EZH2 is upregulated in breast carcinoma cells and is associated with aggressiveness of the disease $\mathrm{e}^{20}$.

Here in this study, we showed close association of EZH2 and NIC-induced increased breast cancer progression. Samples from smoking and never-smoked breast cancer patients, cell lines and xenografts were assessed for EZH2 expression level and its functional role in response to NIC in breast cancer pathogenesis. Using EZH2 inhibitor DZNepA and EZH2si in NIC-treated breast cancer cells, involvement of EZH2 in NIC-mediated aggressiveness of the disease was evaluated. An increased tumor uptake was observed in the xenograft mice model upon NIC treatment. Also, our in vivo studies demonstrated the efficacy of DZNepA in reducing the tumor burden. Using Bupropion, an NIC antagonist, we validated the NICinduced Myc-mediated EZH2 expression. Increased fold enrichment of Myc on EZH2 promoter upon NIC treatment as obtained by CHIP-qPCR strengthened the involvement of Myc in NIC-mediated enhanced EZH2 expression. Overall, we show that NIC exposure either through smoking in breast cancer patients or upon direct treatment in vitro and in vivo resulted into an increased EZH2 expression in breast cancer cells.

\section{Results}

\section{Breast cancer patient samples from smoking individuals} harbor enhanced EZH2 expression

Six pairs of breast cancer tissue (biopsy) from smokers or non-smokers were included in the study. All the cases were carefully selected and compared based on age of the breast cancer patients, the minimum stage of the disease based on grade and histological similarity of the tumor as EZH2 expression is associated with aggressiveness of the disease. Pairs of never-smoked and smoking-associated patient samples $1 \mathrm{Aa}$ and $1 \mathrm{Ab}, 1 \mathrm{Ag}$ and $1 \mathrm{Ah}$, and $1 \mathrm{Ak}$ and $1 \mathrm{Al}$ belonging to similar stage of disease and tumor grade displayed increased EZH2 expression in smoking ones. In one of the pair of samples, non-smoking sample $1 \mathrm{Ae}$ is from more advanced stage (metastatic adenocarcinoma with a higher tumor grade 3) whereas the smoking tumor tissue sample $1 \mathrm{Af}$ is from a comparatively less aggressive breast cancer (non-metastatic and tumor grade 2), but EZH2 expression is still significantly high in cancer tissue from smoking individual. In another pair of samples $1 \mathrm{Ai}$ and $1 \mathrm{Aj}$, both are of stage IIIC; the non-metastatic breast tumor associated with smoking harbor higher EZH2 expression in comparison to non-smoking counterpart, which is from more aggressive, i.e. metastatic breast tumor. Although higher EZH2 expression harboring smoking-associated sample $1 \mathrm{Ad}$ is from higher-grade tumor, the sample $1 \mathrm{Ac}$ from never-smoked individual is a metastatic adenocarcinoma. The overall analysis of clinical samples for EZH2 expression level among smokers and non-smokers led us a hypothesis that EZH2 might have some role in NIC-mediated breast cancer progression (Fig. 1a-d).

\section{NIC treatment results into enhanced EZH2 expression in breast carcinoma cells and abrogates the effect of DZNepA or EZH2si}

The association of NIC with a high level of EZH2 was confirmed in vitro in both cancerous and non-cancerous breast cancer cell lines. To validate our hypothesis that EZH2 plays a significant role in NIC-mediated increased breast cancer progression without limiting to specific cell line, we included one normal breast epithelial cell line MCF-10A, two estrogen receptor positive cell lines T47D and MCF-7, and two aggressive estrogen receptor negative breast cancer cell lines MDA-MB-231 and MDA-MB453 in the study where we found similar kind of results. NIC treatment on normal breast epithelial cells and breast carcinoma cells resulted in the overexpression of EZH2 (Fig. 1e) as detected by western blot assay. The high expression level of EZH2 was also confirmed by quantitative reverse transcriptase-polymerase chain reaction (qRT PCR) (Fig. 1f). The upregulation of EZH2 was associated with strong promoter activity upon NIC treatment as revealed from luciferase assay (Fig. 1g). As already reported, DZNepA treatment depletes EZH2 through increased proteasome-mediated degradation or accumulation of strong product inhibitor $S$-adenosyl homocysteine $^{21}$; we checked the effect of NIC on EZH2 expression post-DZNepA treatment where NIC was able to abrogate the effect of DZNepA (Fig. 1h). At the same time, NIC inhibited the effect of EZH2si on EZH2 expression. (Fig. 1i).

\section{Inhibition of EZH2 by DZNepA or EZH2si reduces NIC-} induced breast cancer progression

Increasing concentration of NIC in MDA-MD-231 and T47D cell lines showed significant increase in the number 


\section{A}

Breast tumor tissues, EZH2 Immunohistochemistry
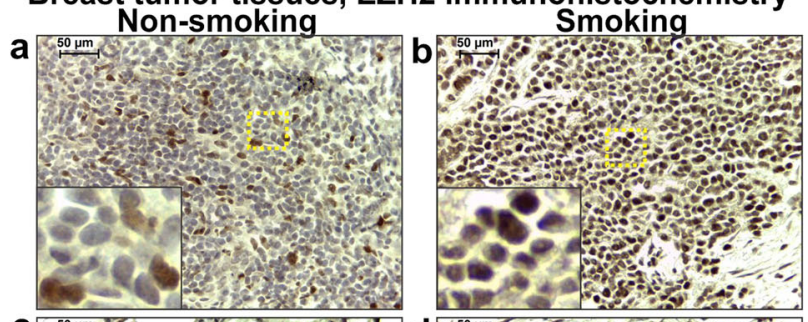

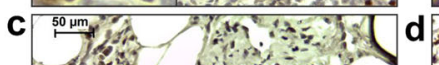
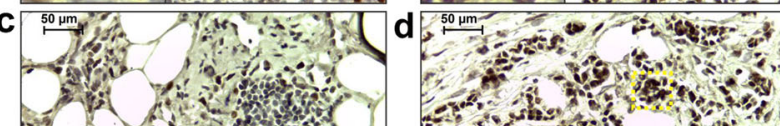

(2)

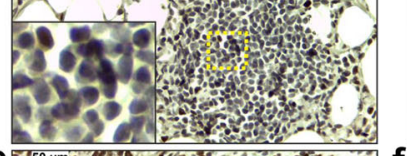

e

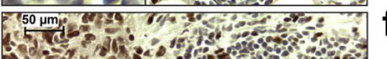

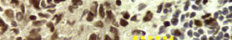

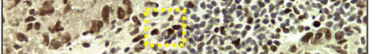

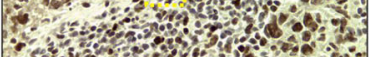

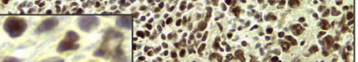

-7.

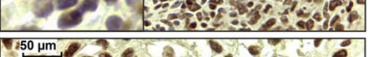

g
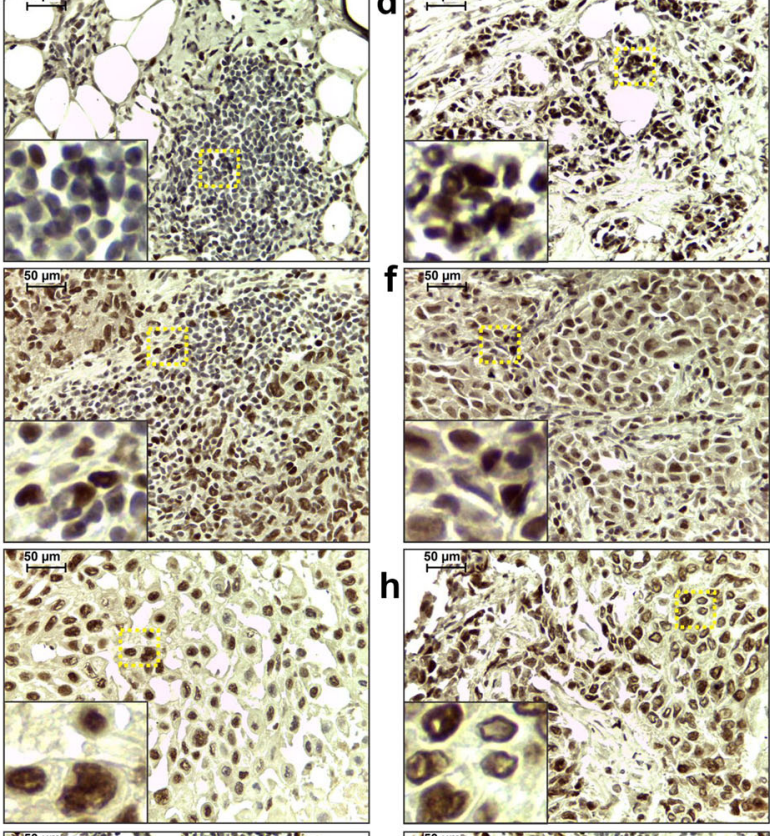

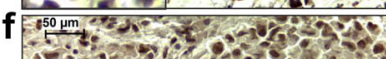

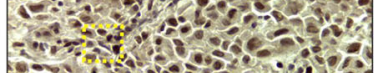

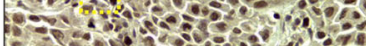

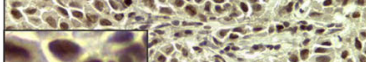

- 20 .

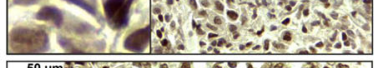

$\mathbf{h}$

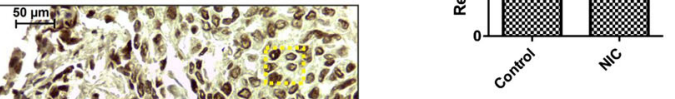

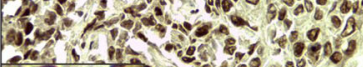
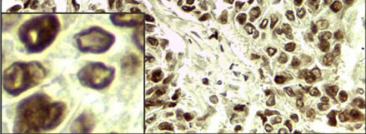

j

I)
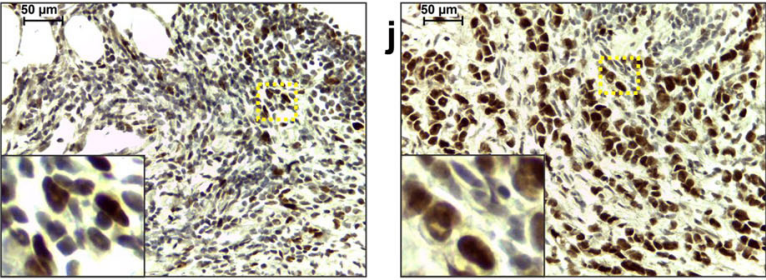

$k$ tom
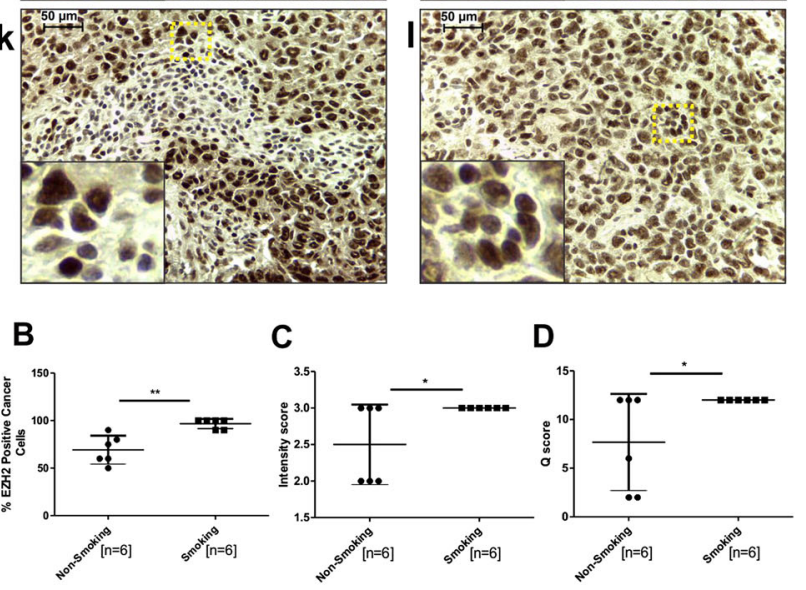
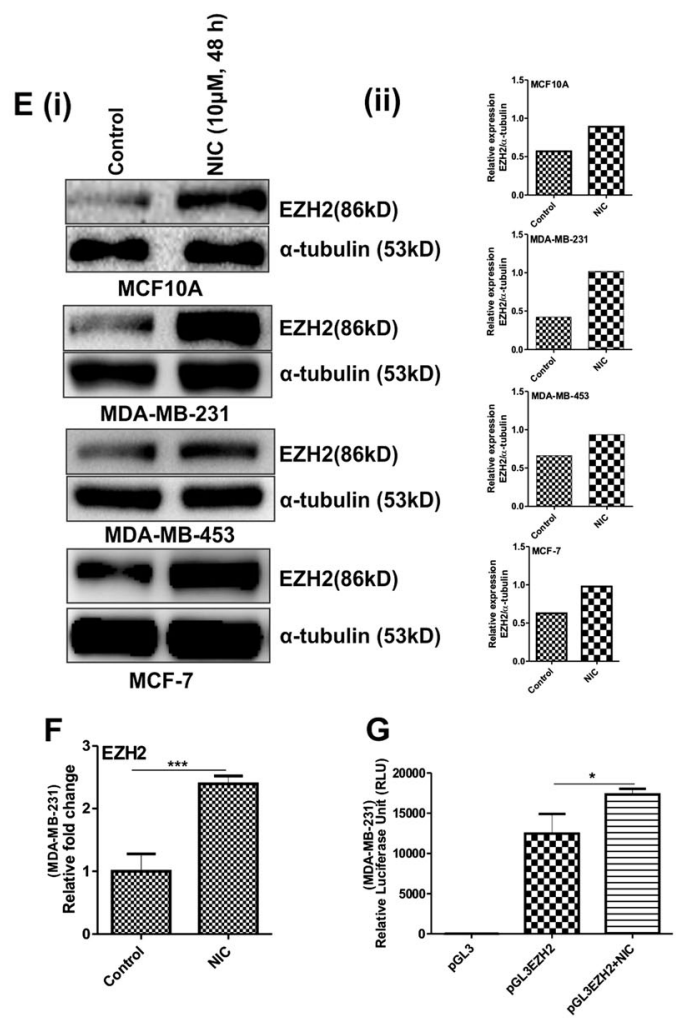

H (i)
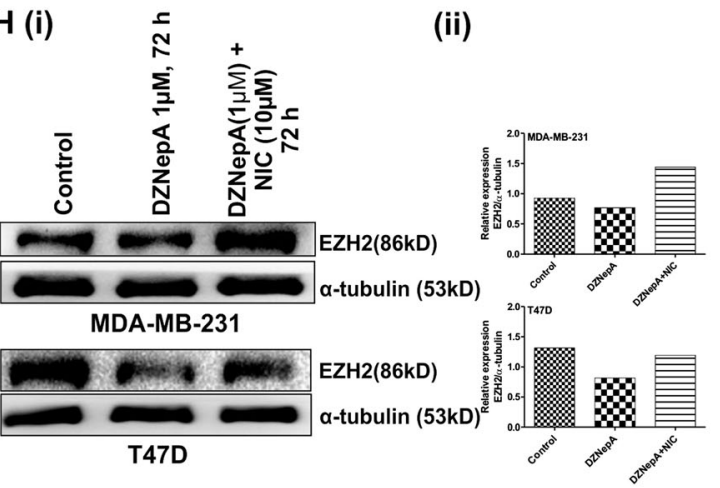

I (i)

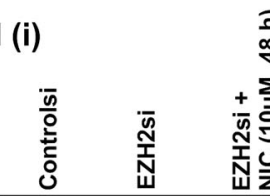

(ii)

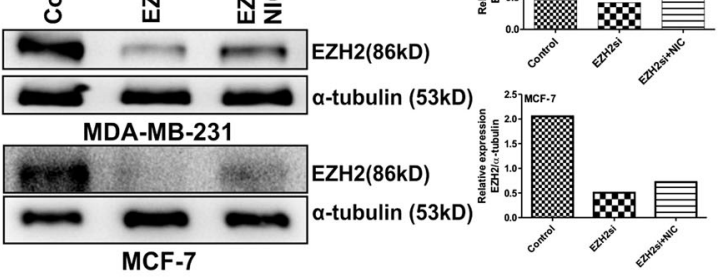

Fig. 1 (See legend on next page.) 


\begin{abstract}
(see figure on previous page)
Fig. 1 NIC exposure either through smoking in breast cancer patients or upon direct treatment in breast cancer cells results into increased EZH2 expression. a Immunohistochemical stained breast cancer patient samples from smoking and non-smoking individuals showing the difference in $\mathrm{EZH} 2$ expression. Insets show 10 times digitally magnified pictures of images captured with $\times 40$ objective. $\mathbf{b}$ Scatter plot shows the percentage of EZH2-positive cancer cells in both the groups. c Difference in the intensity score of EZH2 expression in two groups is depicted in the graph. d Graph represents the $Q$ score for EZH2 expression in tissue sections of smoking and never-smoked breast cancer patients. e (i) Western blot shows upregulated EZH2 expression upon NIC treatment in breast cancer cell lines including normal breast epithelial MCF-10A cells. e (ii) Quantification of western blot was done using software ImageJ. $\mathbf{f}$ Graph displays the relative fold change of EZH2 expression at mRNA level between control and NIC-treated MDA-MB-231 cells. $\mathbf{g}$ Increased EZH2 promoter activity was observed in luciferase assay upon NIC treatment in cancer cells. $\mathbf{h}$ (i), i (i) Immunoblot showing the depleted EZH2 protein level in MDA-MB-231 breast cancer cells upon DZNepA treatment and after EZH2si transfection, respectively, which was recovered following NIC treatment. $\mathbf{h}$ (ii), i (ii) Graphs showing normalized EZH2 expression calculated with respect to a-tubulin. Graphs are plotted with SD, which is calculated from indicated number of samples used for the study. Two-tailed paired Student's $t$-test and one-way ANOVA was used for statistical analysis. ${ }^{*} P<0.05,{ }^{* *} P<0.005,{ }^{* *} P<0.001$. Scale bar $50 \mu \mathrm{m}$
\end{abstract}

of viable cells. Simultaneous treatment of $1 \mu \mathrm{M}$ DZNepA in cells treated with varying concentrations of NIC resulted in notable reduction in the number of viable cells (Fig. 2a). Similarly, in cells treated with varying NIC concentrations, a notable decrease in the number of viable cells was observed upon EZH2si transfection (Fig. 2b). MTT assay performed with varying concentrations of DZNepA significantly reduced the number of viable cells. In comparison to only DZNepA-treated cells, a subsequent significant increase in the percentage of viable cells was found in cells co-treated with $10 \mu \mathrm{M} \mathrm{NIC} \mathrm{and}$ respective increasing concentration of DZNepA, which indicated the inhibitory effect of NIC on DZNepA (Fig. 2c). Increased cell migration upon NIC treatment was found to be inhibited by DZNepA (Fig. 2d). Further, a notable increase in mesenchymal markers and a decrease in epithelial cell markers in NIC-treated cells was found to be reversed in DZNepA and NIC co-treated cells at both protein and mRNA level (Fig. 2e, f). Role of EZH2 in epithelial to mesenchymal transition (EMT) is well known. To investigate the role of NIC in modulating EMT markers by elevating the level of EZH2 in cancer cells, the cancer cells were first treated with NIC for $72 \mathrm{~h}$ and then the NIC-treated cells were transfected with controlsi or EZH2si (in absence of NIC). After $24 \mathrm{~h}$ of transfection, cells were again treated with NIC for another $48 \mathrm{~h}$. Immunoblot showed increased EZH2 level and altered expression of proteins involved in EMT in NIC-treated cells, which was inhibited upon EZH2 knocked-down, thus signifying the fact that NIC modulates the EMT markers in an EZH2 dependent manner (Fig. 2g). Also, NIC-induced increased cell invasion was checked upon DZNepA treatment (Fig. 2h) and EZH2si transfection (Fig. 2i). Apart from the role of VEGF in angiogenesis, it also increases cancer cell growth, migration, and inva$\operatorname{sion}^{22}$. Also, it is involved in key aspects of tumorigenesis such as tumor initiation ${ }^{23}$. Cancer cell growth and invasion is affected by altered expression of matrix metalloproteinase $2^{24}$. Further, to validate the previously reported
NIC-mediated increased levels of VEGF ${ }^{25}$ and MMP- $2^{26}$, we checked their expression in MDA-MB-231 upon NIC/ DZNepA treatment. A reduced expression of VEGF and MMP-2 expression was observed in DZNepA and NIC co-treated cells in comparison to only NIC-treated cells, which emphasizes on the role of NIC on cell invasion property through EZH2 (Fig. 2j). DZNepA is reported to induce apoptosis in cancer cells ${ }^{21}$; on the other hand, NIC provides survival advantage to the cells by making them resistant to apoptosis. More than 10\% reduction in early apoptotic cells were observed in cells simultaneously treated with NIC and DZNepA (Fig. 2k). Apoptosis is regulated in part by $\mathrm{Bcl}-2$ genes that regulate cell survi$\mathrm{val}^{27}$. Reduced apoptosis and neuroprotective effect of NIC is marked by reduced cleavage of PARP- $1^{28,29}$. NIC treatment resulted into a significant increase in Bcl-2 expression that reduced upon DZNepA treatment. Additionally, reduced PARP cleavage in cells co-treated with NIC and DZNepA in comparison to only DZNepAtreated cells corroborated the protective effect of NIC on DZNepA-induced apoptosis (Fig. 2l). These data showed the effect of DZNepA and EZH2si on NIC-induced enhanced breast cancer cell progression and thus indicated the direct involvement of EZH2.

\section{NIC treatment led to increased tumor uptake in xenograft} nude mice model and upon DZNepA treatment, NICinduced increased tumor growth was significantly reduced

To understand the role of NIC in increasing the breast cancer risk, the xenograft nude mice model was used. Twenty-four mice were involved in the study for 11 weeks (Fig. 3a). We considered $100 \mathrm{~mm}^{3}$ tumors size as cut-off volume for a well-established tumor. After 60 days of treatment, among 12 vehicle-treated mice, only 1 developed and established the tumor. However, out of 12 NICtreated mice, 10 mice developed the tumor, which was growing well. This suggested that NIC increases the tumor uptake by $75 \%$ (Fig. 3b) and thus might be a strong risk factor for breast cancer. Role of survivin in malignant 


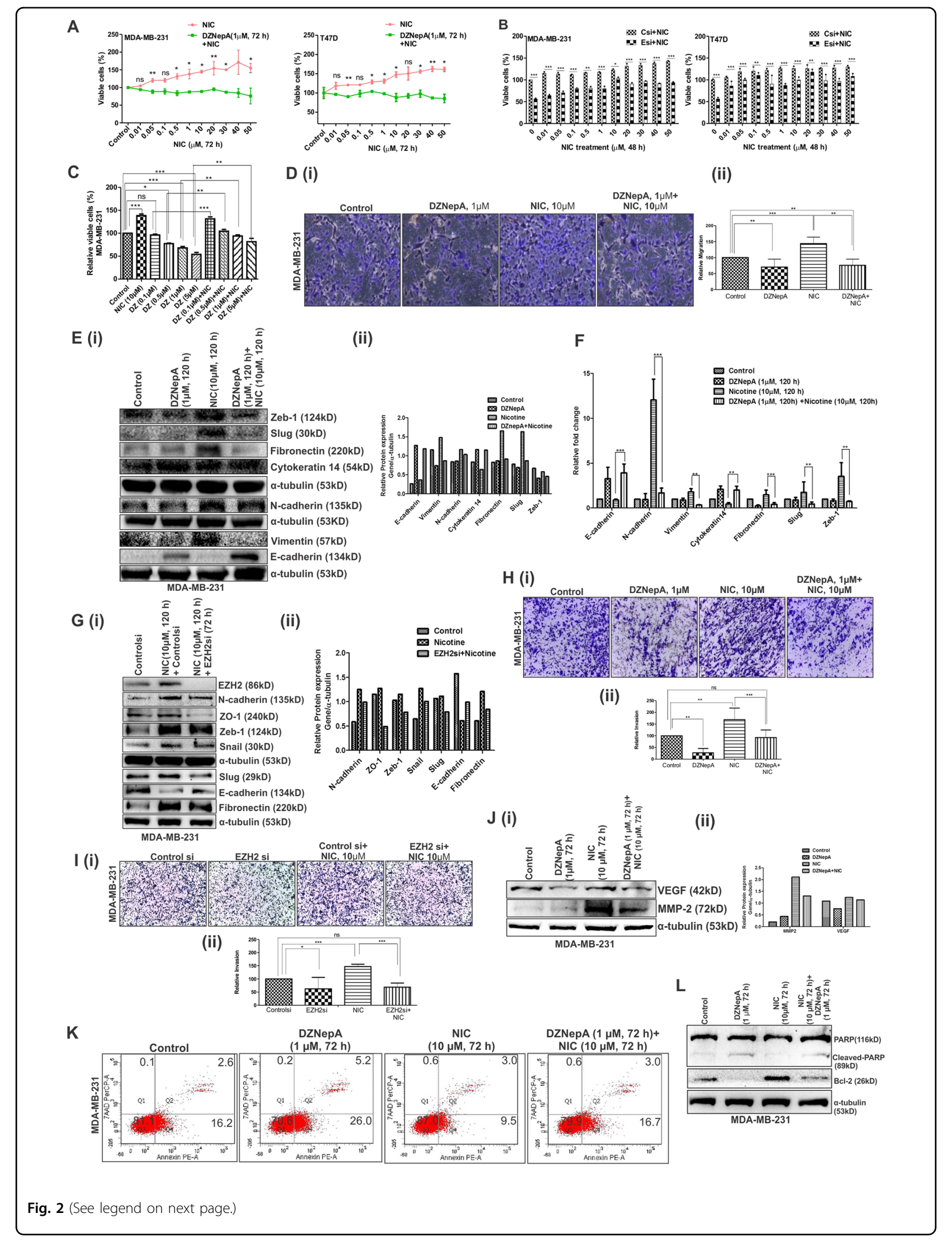




\begin{abstract}
(see figure on previous page)
Fig. 2 Transfection of EZH2si or treatment of DZNepA in NIC-treated breast carcinoma cells led to reduced breast cancer progression. a MTT assay shows the antagonistic effect of DZNepA on NIC-mediated increased number of viable cells in MDA-MB-231 and T47D breast cancer cells. b Graph illustrates number of viable cells upon EZH2si transfection in NIC-treated cells. c Effect of varying concentrations of DZNepA or co-treatment of NIC and DZNepA on number of viable cells after $72 \mathrm{~h}$ of treatment. $\mathbf{d}$ (i) Picture showing $\times 20$ images of transwell migration assay performed to study the effect of DZNepA on NIC-mediated increased cell migration. $\mathbf{d}$ (ii) Graph depicts the relative percent of migration in all four experimental groups in comparison to control groups. e (i), $\mathbf{g}$ (i) Expression of proteins involved in epithelial to mesenchymal transition (EMT) upon NIC/DZNepA/ EZH2si treatment/transfection. e (ii), $\mathbf{g}$ (ii) Normalized protein expression as observed in western blots. $\mathbf{f}$ qRT PCR shows the expression of EMT-related genes at mRNA level. $\mathbf{h}$ (i), i (i) Representative pictures $(\times 20)$ of invasion assay demonstrates the inhibitory effect of DZNepA and EZH2si, respectively, on NIC-induced increased invasion of cancerous cells. $\mathbf{h}$ (ii), $\mathbf{i}$ (ii) Graph shows the relative percent of invasion compared to control in the experimental groups for experiments done in triplicate. $\mathbf{j}$ (i) Immunoblot displaying the effect of DZNepA on NIC-induced increased MMP-2 and VEGF expression. $\mathbf{j}$ (ii) Bar diagram shows normalized protein expression. $\mathbf{k}$ The effect of NIC on DZNepA-mediated increased apoptosis in MDA-MB-231 cells is evident from the flow cytometry data. I Difference in the expression of $\mathrm{BCl}-2$ and Cleaved-PARP in different experimental groups is shown in the western blot. Graphs are plotted with SD, which is calculated from three independent experiments. Two-tailed paired Student's $t$-test, one-way and two-way ANOVA were used for statistical analysis for experiments done in triplicate. ${ }^{*} P<0.05,{ }^{* *} P<0.005,{ }^{* * *} P<0.001$
\end{abstract}

transformation of normal human bronchial epithelial cells is previously reported ${ }^{30}$. To validate that NIC provides survival advantage to cancer cells ${ }^{28,}{ }^{31}$, we checked the expression of survivin upon NIC treatment both in vitro and in vivo. In response to NIC treatment, expression of survivin was upregulated in both xenograft and MDAMB-231 breast cancer cells (Fig. 3c), thus supporting the proposal that NIC provides survival benefit to the cancer cells which led to increased tumor uptake in mice model. Upon DZNepA treatment in NIC-treated mice, the mean tumor volume was reduced by $74 \%$ (Fig. 3f). However, the xenografts of NIC-treated mice grew exponentially.

\section{Enhanced expression of EZH2 and $\mathrm{Ki} 67$ in xenografts from} NIC-treated mice was reduced upon DZNepA treatment

Similar to the results observed in smoking patient samples, immunohistochemistry in tumor sections of NIC-treated $(0.25 \mathrm{mg} / \mathrm{kg}$ body weight, 80 days $)$ mice showed 30\% enhanced EZH2 expression (Fig. 4a, b) . At the same time, expression of proliferation marker Ki67 was upregulated by $22.5 \%$ in NIC-treated tumor samples (Fig. 4a, c). Upon DZNepA treatment $(1 \mathrm{mg} / \mathrm{kg}$ body weight, 20 days), expression of EZH2 was reduced by $48 \%$ and a reduction of $36.5 \%$ was observed in the expression of Ki67, which clearly indicated the potential of DZNepA in reducing the tumor burden. A similar trend of EZH2 expression was also observed in protein extracted from vehicle, NIC, and NIC and DZNepA co-treated xenografts by western blot assay (Fig. 4d).

NIC dependent enhanced EZH2 expression is induced by increased expression of Myc in response to NIC exposure

Myc is a transcription factor, which positively regulates EZH2 by directly binding on the E-box regulatory promoter region ${ }^{32}$. Positive correlation exists between EZH2 and Myc in breast cancer cells (Fig. 5a) as well as in primary breast tumor (Fig. 5b) as computed from the MERAV expression datasets ${ }^{33}$. In response to NIC, an increased expression of $\alpha 9-\mathrm{nAChR}$, Myc, and EZH2 was observed in vitro (Fig. 5c) and in vivo (Fig. 5d). Upon treatment with the NIC antagonist Bupropion, the effect of NIC was reversed and reduced expression of EZH2 and Myc was observed in MDA-MB-231 cells (Fig. 5e). CHIPqPCR showed about two-fold enrichment of Myc at E-box element (CACGTG) on EZH2 promoter in NIC-treated MDA-MB-231 cells (Fig. 5f, g). These data suggested the possible mechanism behind the enhanced EZH2 expression in response to NIC in breast cancer and thus in increased disease progression (Fig. $5 \mathrm{~h}$ ).

\section{Discussion}

Tumor-promoting activity of NIC is reported in various types of cancers. Upon activation by NIC, nAChR activate several growth stimulating signaling pathways. In breast cancer, activation of specific $n A C h R \alpha-9$ is reported during transformation of normal breast epithelial cells. Although NIC is studied for its effect on non-neuronal cells, such as cancer cells, association of NIC-dependent epigenetic alteration with cancer pathogenesis is not known. In this study, we demonstrated that NIC-induced increased breast cancer progression is mediated through EZH2 and enhanced EZH2 expression in response to NIC is primarily through Myc upregulation. As NIC is one of the constituents of cigarette smoke, degree of NIC exposure in the smoking individuals and the corresponding effect on EZH2 expression is debatable. However, in in vitro cell culture, enhanced expression of EZH2 was detected even at nanomolar concentrations within $24 \mathrm{~h}$ of NIC treatment (Figure S2). Our luciferase assay data (Fig. 1g) indicated the involvement of transcription factors in NICmediated increased EZH2 expression. EZH2 is regulated by molecules such as Myc, E2F, Sox4, Elk1, HIF1, etc. ${ }^{34}$. Apart from directly binding to E-box element of EZH2 promoter, Myc also regulates EZH2 expression by epigenetically repressing ${ }^{35}, 36$ the negative regulators miRNA-101 and miRNA-26a ${ }^{37,38}$. miRNA-101 is reduced 
upon NIC treatment ${ }^{39}$. In a transcriptome study, Myc was found to be upregulated upon NIC treatment ${ }^{40}$.

To investigate the correlation between EZH2 and Myc, we analyzed MERAV gene expression dataset that is designed to analyze human gene expression across large variety of arrays (4454 arrays). The Pearson correlation coefficient values computed using its breast cancer cell line and patient sample datasets are 0.02 and 0.22 , respectively (Fig. 5a, b). The values indicate that Myc and EZH2 shares a positive correlation, which is not very strong. The possible reason for which may be that only one transcription factor Myc may not be sufficient to regulate EZH2 in different heterogeneous tumors/cell lines with a diverse genetic background. The genetic diversity might be playing a role in regulation of EZH2 in association with Myc. Bupropion treatment resulted into reduced $\alpha 9$-nAChR, Myc, and EZH2 expression, indicating receptor-mediated upregulation of Myc and EZH2 in response to NIC treatment.

EZH2 expression is associated with tumor grade and aggressiveness of the disease. Higher is the tumor grade, more is the expression of EZH2. More aggressive is the disease, higher is the EZH2 expression. We were unfortunate to get the samples from lower grade of tumor such as grade 1 where probably the difference could be more distinct between smoking and non-smoking samples; however, even in higher grade of tumors, a notable difference was found in the EZH2 expression irrespective of the tumor grade and histological difference. The samples under study majorly display alternate combinations of breast ductal adenocarcinoma and lobular adenocarcinoma among never-smoked and smoking-associated breast cancer patients. Furthermore, the samples from never-smoked patients harbor low level of EZH2 irrespective of ductal or lobular type of cancer tissue in comparison to smoking-associated breast cancer tissue samples, which minimizes the role of inter-tumoral heterogeneity in EZH2 expression level.

In vitro data indicated increased EZH2 as a consequence of NIC treatment, which was strengthened by analyzing the effect of NIC post-DZNepA and EZH2si action. NIC is well studied for its immune suppressive role ${ }^{41-44}$ in humans. NIC and DZNepA treatment did not have significant effect on immune cell numbers in nude mice (Figure S1) as observed in Exigo veterinary hematology system. This suggested that the dosage used in the study for NIC and DZNepA did not affect the immune cells number. The body weights of the mice were found to be unaffected in the treatment groups, which also indicated that treatments did not have any adverse effect on the health of the animal.

The anatomical location has a significant influence on overall xenograft success ${ }^{45}$. The most appropriate site routinely used for breast tumor xenografts is mammary fat pad. We chose xenograft nude mice model to study the effect of NIC on tumor aggressiveness/implantation by modulating EZH2 expression and role of DZNepA in reducing NIC-induced increased tumor burden. One hundred percent tumor uptake is reported with MDAMB-231 in the mammary fat pad (m.f.p.) injections whereas only $40 \%$ of the subcutaneous injections are reported to produce tumors several weeks after the appearance of the m.f.p. tumors ${ }^{46,47}$. In our study, among 12 vehicle-treated mice, 4 (about 33.33\%) showed up with tumors initially (Figure S3 showing the picture of three mice with a tumor size less than $100 \mathrm{~mm}^{3}$ ), but with time in only one mice, tumor of proper size $\left(100 \mathrm{~mm}^{3}\right)$ was successfully established and grew slowly compared to NIC-treated groups. In literature, different immunedeficient mouse strains have been used for establishing experimental tumors; however, in many instances their genetic background and source have not been clearly mentioned. Studies have evidently shown a distinct variability in establishing xenografts in different strains of immune-deficient mouse and this could be due to their differential residual adaptive and innate immunity present in these mice ${ }^{48}$. At the same time, the unavoidable variation in nude mice rearing and handling in different animal facilities might also contribute to the overall residual immune status of these mice. Taken together, we believe that the low percentage of tumor uptake in the vehicle-treated group of animals might be due to any of these possibilities.

Pierce et al. (2013) ${ }^{40}$ showed breast cancer deaths, recurrence, and survival proportions in never-smokers, former smokers, and current smokers, providing evidence to support NIC as a potent risk factor for breast cancer. Increased breast cancer deaths, recurrence, and reduced survival of current smokers indicate the role of NIC the most common constituent of cigarette smoke in providing survival advantage to the cancer cells. In support of the above study, our xenograft model showed higher uptake of tumor (higher number of xenografts successfully established) in NIC-treated animals in contrast to vehicletreated mice, which corroborated with our in vitro data, suggesting that NIC provides survival advantage to the cancer cells by increasing the rate of proliferation (higher Ki67 positive, high MTT reading in vitro). Increased MTT reading and upregulated Ki67 and survivin expression upon NIC treatment suggest the role of NIC in increased successful tumor implantation.

Treatment with DZNepA in NIC-stimulated tumors resulted in reduced tumor growth, which shows its efficacy as a therapeutic drug in breast cancer treatment. DZNepA is an adenosine homocysteine hydrolase inhibitor that inhibits methyltransferases indirectly ${ }^{49}$. The mechanism underlying the inhibition of EZH2 by DZNepA communicates the involvement of other 
A

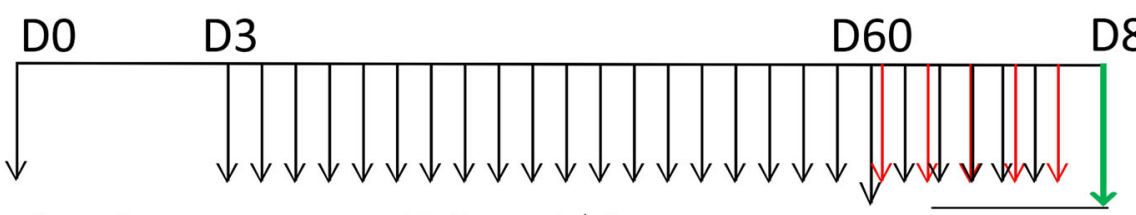

Injection of

MDA-MB-231

Breast

Cancer Cells $\left(10^{7}\right)$

$(n=24)$

B

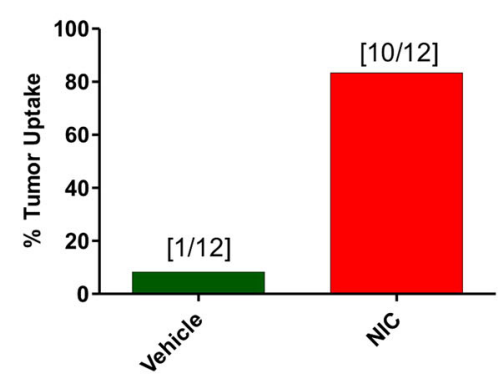

IP, Every $3^{\text {rd }}$ day

$\downarrow$ IP $4^{\text {th }}$ day; $\forall$ IP $3^{\text {rd }}$ day

$\downarrow \mathrm{NIC}(0.25 \mathrm{mg} / \mathrm{Kg}$ body wt) $\quad \downarrow \mathrm{DZNepA}(1 \mathrm{mg} / \mathrm{kg}$ body weight $)+$ ( $\mathrm{n} 1=12)$, Vehicle $(\mathrm{n} 2=12)$

$\downarrow N I C(0.25 \mathrm{mg} / \mathrm{Kg}$ body wt)

(n1'=5), NIC (n1'=5)

C

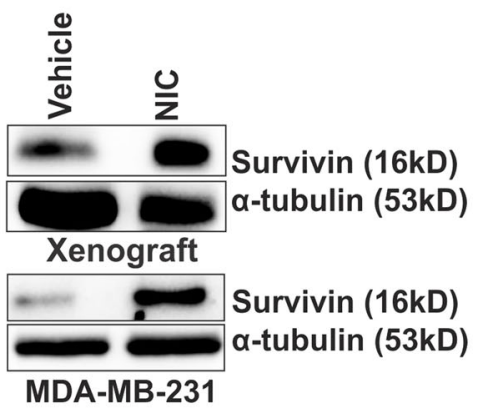

D

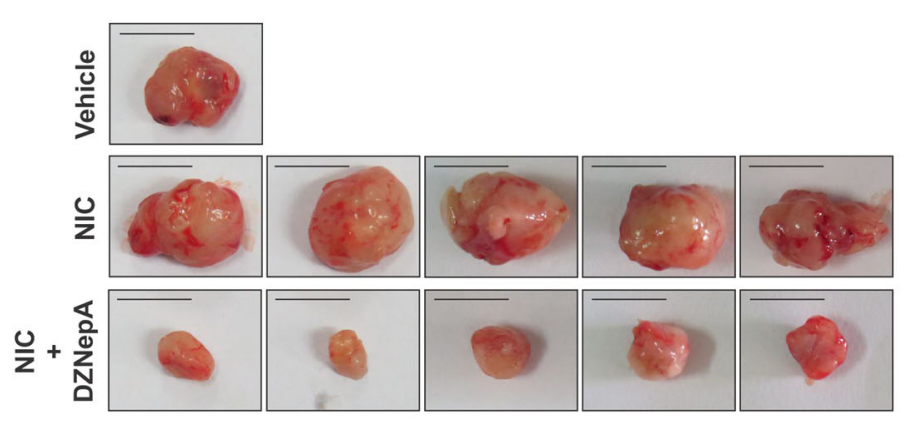

E

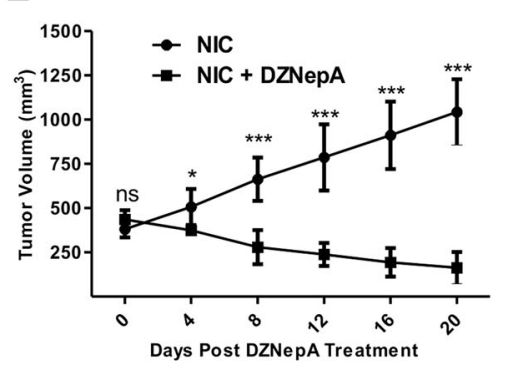

$\mathbf{F}$

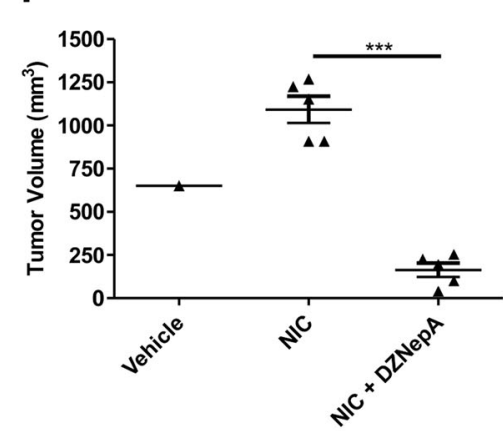

G

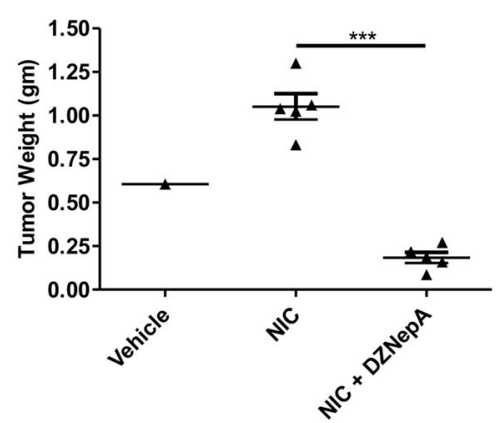

Fig. 3 Increased tumor uptake was found in NIC-treated mice and DZNepA significantly reduced the enhanced tumor growth in response to NIC treatment. a Diagram depicts the overall xenograft study. $\mathbf{b}$ Graph showing percent tumor uptake in vehicle and NIC-treated group of mice. c Immunoblot shows survivin expression in response to nicotine exposure in xenograft and MDA-MB-231 breast cancer cells. $\mathbf{d}$ Picture of tumors collected at the end of the experiment in the three experimental groups. e Graph demonstrates the effect of DZNepA on NIC influenced tumor growth. $\mathbf{f}, \mathbf{g}$ Difference in tumor volume and weight in three experimental groups are illustrated in the graph respectively. Black and red arrow indicates IP injection of NIC and DZNepA, respectively. Green arrow indicates the day of termination of experiment. As a reference, the ruler was used while capturing the digital image of individual tumor and scale bar of $1 \mathrm{~cm}$ is used as a reference for tumor size. Two-tailed paired Student's $t$-test was used for statistical analysis. ${ }^{*} P<0.05,{ }^{* * *} P<0.001$ 


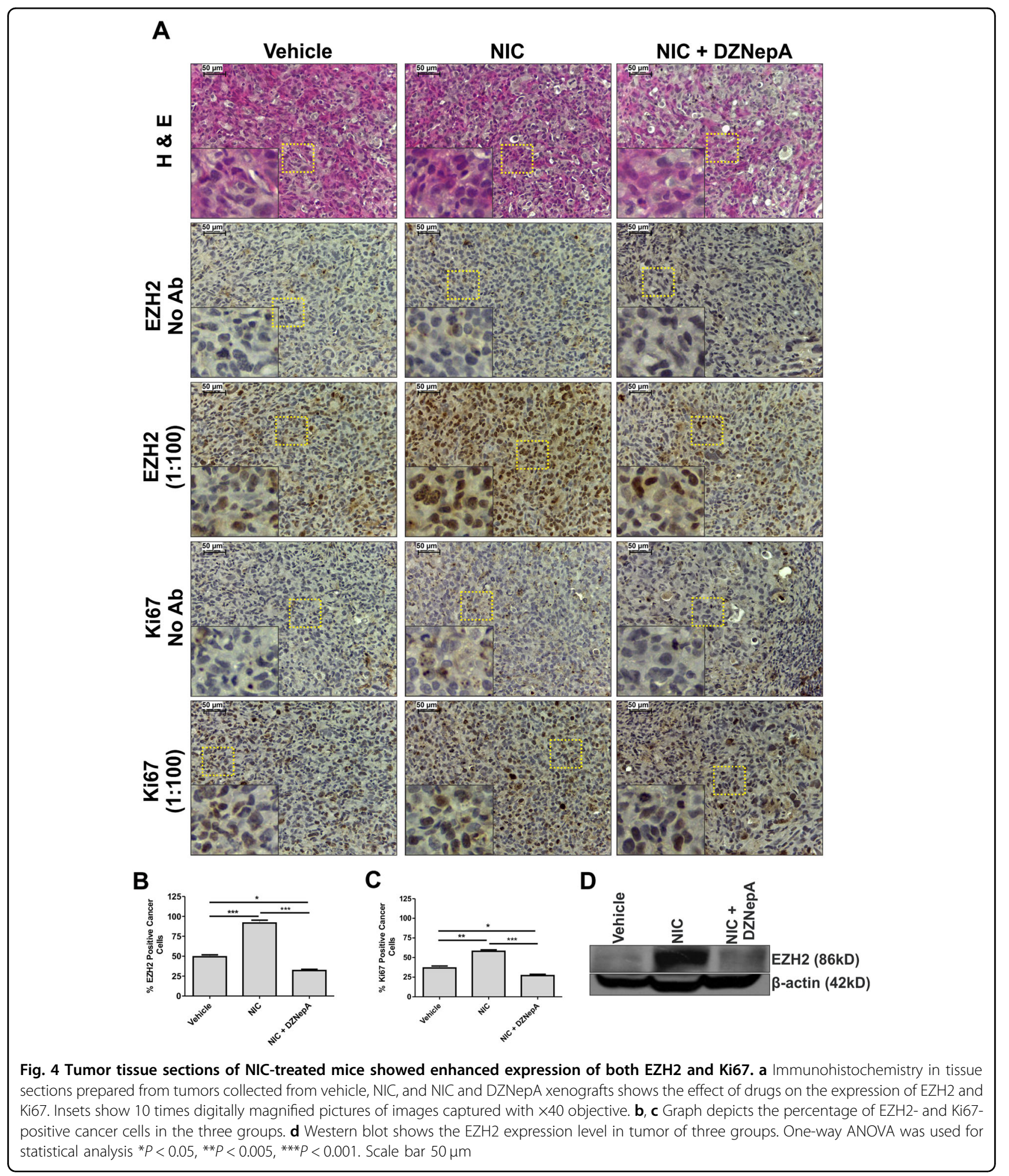

methyltransferases in NIC-mediated increased breast cancer risk, which needs further investigation. In conclusion, we demonstrate that in response to NIC there is an enhanced expression of Myc, which in turn increases
EZH2 expression and results into increased breast cancer progression. Thus, polycomb group protein EZH2 plays a significant role in NIC-mediated increased breast cancer progression. 


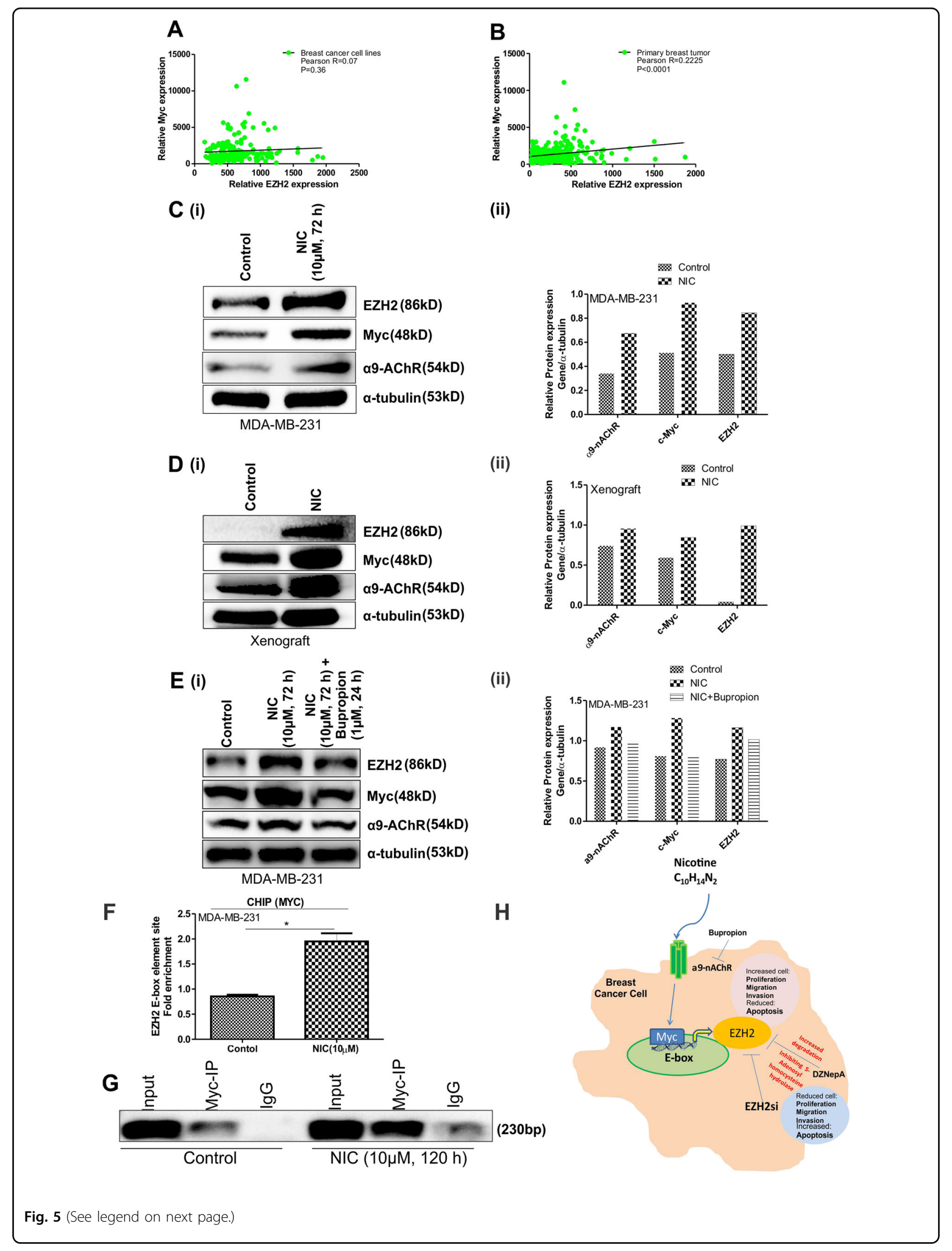




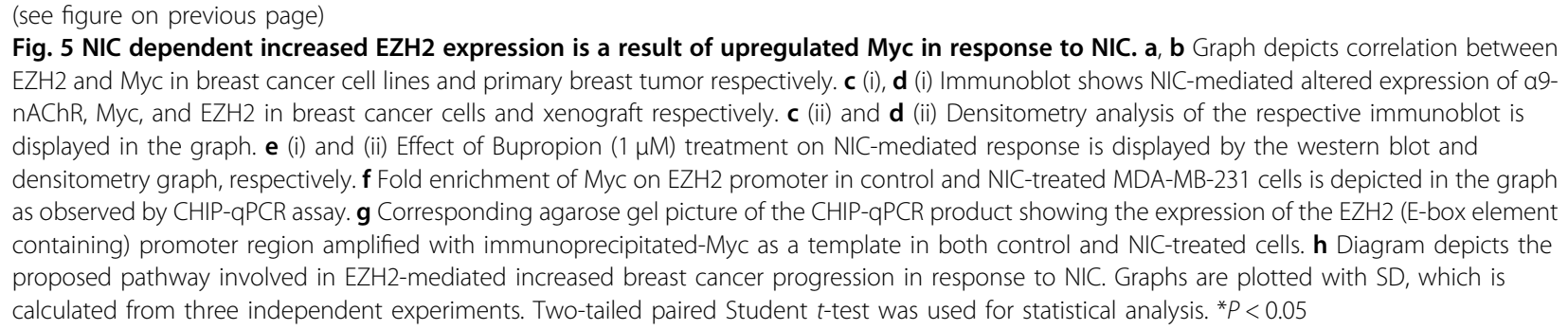

\section{Materials and methods}

\section{Human breast cancer patient samples}

Human breast carcinoma frozen tumor tissue sections from smoking and never-smoked female breast cancer patients used in the study were purchased from a US based company OriGene. Table S1 contains all the details of the patient samples used in the study. Breast cancer patient samples associated with smoking were matched with never-smoked ones depending on age, minimum stage of the disease, and Nottingham tumor grade system.

\section{Cell culture and treatment}

The human breast carcinoma cell lines MCF-7, MDA-MB231, T47D, and MDA-MB-453 were obtained from National Repository of Animal Cell Culture, NCCS Pune (Maharashtra, India) and independently validated by STR DNA fingerprinting at Institute of Life Sciences (Bhubaneswar, India). T47D, MDA-MB-231, and MDA-MB-453 cell lines were maintained in Roswell Park Memorial Institute 1640 (RPMI) medium and MCF-7 was maintained in Dulbecco's modified Eagle's medium (DMEM) containing 10\% fetal bovine serum supplemented with penicillin-streptomycin at $37^{\circ} \mathrm{C}, 5 \% \mathrm{CO}_{2}$, and 95\% humidity. MCF-10A, a kind gift from Dr. Annapoorni Rangarajan (IISC, Bangalore, India), was maintained in DMEM F12 containing horse serum (5\%) supplemented with hydrocortisone $(0.5 \mathrm{mg} / \mathrm{ml})$, EGF $(20 \mathrm{ng} / \mathrm{ml})$, insulin $(10 \mu \mathrm{g} /$ $\mathrm{ml})$, cholera toxin $(100 \mathrm{ng} / \mathrm{ml})$, and penicillin-streptomycin at $37^{\circ} \mathrm{C}, 5 \% \mathrm{CO}_{2}$, and $95 \%$ humidity. For treatment, appropriate concentration of DZNepA or NIC was directly added to the culture dish.

\section{EZH2 promoter assay}

EZH2 promoter (1884 bp) was cloned into pGL3 luciferase vector (Addgene) using specific primers (Table S3). The generated pGL3EZH2 construct was transfected into MDA-MB-231 cells using Lipofectamine 3000 (Invitrogen) as per the manufacturer's protocol. After $24 \mathrm{~h}$ of transfection NIC was treated and further after $48 \mathrm{~h}$ luciferase activity was quantified using Lucifease assay reagent (Promega). The luciferase reading was taken in a luminometer (Sirius, Titertek-Berthold).

\section{In vivo xenograft studies and drug administration}

Institutional Animal Ethical Committee (Institute of Life Sciences, Bhubaneswar, India) approved all animal experiments. Five- to six-week-old BALB/c female nude mice (model NCRNU-F; nomenclature, CrTac: NCrFoxn $1^{\text {nu }}$; genotype-homozygous $\mathrm{sp} / \mathrm{sp}$ ) were used for xenograft study. MDA-MB-231 $\left(10^{7}\right)$ cells in matrigel were subcutaneously injected into the flank of the mice. After 3 days of cancer cell injection, intraperitoneal injection of NIC $(0.25 \mathrm{mg} / \mathrm{kg}$ body weight $)$ or PBS as a vehicle was administered twice in a week. When tumor size was about $400 \mathrm{~mm}^{3}$ in the NIC-treated group, the animals were randomly divided into NIC $(0.25 \mathrm{mg} / \mathrm{kg}$ body weight $)$ or NIC $(0.25 \mathrm{mg} / \mathrm{kg}$ body weight $)+$ DZNepA ( $1 \mathrm{mg} / \mathrm{kg}$ body weight) group and both the groups continued to receive the above-mentioned dose of NIC twice a week. Vehicle/DZNepA was treated every fourth day by intraperitoneal injection. The initial tumor size of both the groups before DZNepA treatment was around $400 \mathrm{~mm}^{3}$ as indicated in the graph (Fig. 3e). Experiment was terminated after 11 weeks when tumor in DZNepA-treated mice was $\leqslant 100 \mathrm{~mm}^{3}$. Tumor volume was measured every fourth day with a digital caliper. The formula for calculation was tumor volume $=1 / 2$ (length $\times$ width $\left.^{2}\right)^{50}$.

\section{Tissue processing and immunohistochemistry}

Tissues were collected and preserved in 10\% formalin. For histopathological analysis, tissues were processed for slide preparation. Immunohistochemistry in slides of both patient samples and xenografts was performed as described $^{51}$. Slides were incubated with primary antibodies EZH2 (1:100) or Ki67 (1:100) overnight at $4{ }^{\circ} \mathrm{C}$ and then subjected to incubation with anti-mouse/rabbit IgG secondary antibody for $1 \mathrm{~h}$. Diaminobenzidine was used to detect the immunoreactivity. Slides were subsequently stained with hematoxylin and processed further. Stained slides were observed under a light microscope (Leica DM500) and images were captured at $\times 40$ magnification. Pathologist scored all the stained slides as previously described ${ }^{52}$. Briefly, the staining intensity of cancerous cells was scored as: absent or 
weak, 1 point; moderate, 2 points; and strong, 3 points. Percentage positive tumor cells were scored as: 0 for percent of cells $<1,1$ for percent of cells between 1 and 10, 2 for percent of cells between 11 and 33, 3 for percent of cells between 34 and 66, and 4 for percent of cells between 67 and 100. $Q$ score was calculated by multiplying intensity score by the score for percentage of EZH2-positive cancer cells.

\section{Cell viability assay}

For cell viability assay, breast cancer cells were seeded at a density of $3 \times 10^{3}$ cells per well in 96 -well plates. NIC and/or DZNepA were added at appropriate concentrations directly into the medium after $12 \mathrm{~h}$ of cell attachment. Cells were incubated for desired time periods. After stipulated time, cell viability was checked by performing 3-(4,5-dimethylthiazol-2-yl)-2,5-diphenyltetrazolium bromide (MTT) assay ${ }^{53}$. The plate was read at a wavelength of $570 \mathrm{~nm}$ in a Varioskan $^{\mathrm{TM}}$ Flash Multimode Reader (Thermo Scientific).

\section{Migration and invasion assays}

Quantitative functional cell migration and invasion assays were performed using Boyden's chambers consisting of polycarbonate filters with a pore size of $8 \mu \mathrm{m}$ as previously described ${ }^{54}$. Appropriately NIC $(10 \mu \mathrm{M}, 120 \mathrm{~h})$ or DZNepA $(1 \mu \mathrm{M}, 120 \mathrm{~h})$ treated or siRNA transfected MDA-MB-231 cells were processed for the assay. After $24 \mathrm{~h}$, cells migrated to the lower surface were fixed with $10 \%$ formalin and stained with $0.01 \%$ crystal violet stain. After washing, cells migrated towards the serum were observed under a Leica inverted microscope (ICC50 HD), pictured $(\times 20)$ and counted in 10 different random field views. For invasion assay, the upper chamber of transwell was coated with a final concentration of $1 \mathrm{mg} / \mathrm{ml}$ of matrigel in serum-free growth medium.

\section{Western blot}

Appropriately treated cells or xenografts were lysed in RIPA lysis buffer [20 mM Tris- $\mathrm{HCl}$ (pH 7.5) $150 \mathrm{mM}$ $\mathrm{NaCl}, 1 \mathrm{mM} \mathrm{Na} \mathrm{N}_{2}$ EDTA, $1 \mathrm{mM}$ EGTA, $1 \%$ triton $\mathrm{X}, 1 \%$ sodium deoxycholate, $2.5 \mathrm{mM}$ sodium pyrophosphate, 1 $\mathrm{mM} \beta$-glycerophosphate, $1 \mathrm{mM} \mathrm{Na} \mathrm{VO}_{4}$, and $1 \mu \mathrm{g} / \mathrm{ml}$ protease inhibitor] and electrophoresed on 10\% SDSpolyacrylamide gel. The proteins were transferred onto polyvinylidene difluoride (PVDF) membrane. After blocking in 5\% skimmed milk the membrane was incubated with primary antibodies overnight (details of all antibodies and reagents are provided in Table S2). The membrane was then washed with TBS-T and incubated with anti-rabbit or anti-mouse horseradish peroxidase conjugated secondary antibody for $1 \mathrm{~h}$. After washing, the blots were developed using luminol in Chemi-doc (Bio-Rad).

\section{Real-time PCR}

Total RNA from treated breast cancer cells was extracted with Trizol as previously described ${ }^{55}$. Equal amount $(1 \mu \mathrm{g})$ of RNA was used to synthesize cDNA of respective treated samples using cDNA synthesis kit following the manufacturer's protocol. Quantitative realtime PCR was performed on the Roche platform. Details of primers are provided in Table S3. The relative mRNA level or fold change for each gene compared to control was calculated using the value of cycle threshold. Glyceraldehyde 3-phosphate dehydrogenase was used for normalization.

\section{siRNA transfection}

For knockdown experiments, siRNA transfection was done using Lipofectamine 3000 transfection reagent according to the manufacturer's instructions in appropriately treated MDA-MB-231 breast cancer cells. Sequence of siRNA duplexes is provided in Table S4. All experiments involving EZH2si transfection were done within $72 \mathrm{~h}$.

\section{Annexin $\mathrm{V}$ apoptosis assay}

Appropriately treated MDA-MB-231 $\left(2 \times 10^{5}\right)$ cells were processed for apoptosis study using the Annexin $\mathrm{V}$ binding assay as per the manufacturer's instructions. The cells were then analyzed using the FACS system (Becton Dickinson, USA).

\section{Online dataset}

To investigate the correlation between EZH2 and MYC, we used online Metabolic gEne RApid Visualizer (MERAV) database. MERAV is designed to analyze normalized human gene expression across 4454 arrays. Our study includes 196 different established and patient derived breast cancer cell line and 332 primary breast tumors of different grades and histology types available in the dataset.

\section{Chromatin immunoprecipitation}

ChIP assays were performed manually as per the cold spring harbor protocol ${ }^{56}$. Equal amounts of crosslinked DNA were used for Myc or control rabbit IgG. Input and immunoprecipitated DNA was purified using phenolchloroform isoamyl alcohol and ethanol as described ${ }^{57}$. All samples were analyzed by qRT-PCR and were carried in duplicate with primers specific (Table S3) to Mycbinding region (E-box element) on EZH2 promoter. The fold enrichment was determined using the formula: Fold enrichment $=2^{(\Delta C T \text { of input }-\Delta C T \text { of Immunoprecipitated DNA }) 56}$.

\section{Statistical analyses}

Throughout the current study, two-tailed paired Student's $t$-test, one-way and two-way ANOVA was 
performed to test the statistical significance of differences between the experimental groups using the software GraphPad Prism v5.01. Differences in data with values of ${ }^{*} P<0.05,{ }^{* *} P<0.005$ and ${ }^{* * *} P<0.001$ were considered statistically significant.

\section{Acknowledgements}

We acknowledge DBT, Govt. of India and DST, Govt. of India, for funding. We also acknowledge the Director, Institute of Life Sciences, for the core grant as well as his support in the performance of this project.

\begin{abstract}
Author details
${ }^{1}$ Cancer Biology Laboratory, Department of Gene Function and Regulation, Institute of Life Sciences, Bhubaneswar, Odisha, India. ${ }^{2}$ Utkal University, Bhubaneswar, Odisha, India. ${ }^{3}$ Tumor Microenvironment and Animal Models Laboratory, Department of Translational Research, Institute of Life Sciences, Bhubaneswar, Odisha, India. ${ }^{4}$ Manipal University, Manipal, Karnataka, India. ${ }^{5}$ Pathology Department, Hemalata Hospital, Chandrashekharpur Bhubaneswar, Odisha, India. ${ }^{6}$ University of Nebraska Medical Center, Nebraska NE USA
\end{abstract}

\section{Author contributions}

Study conception and design: K.K., B.D., S.C., and S.K.M. Acquisition of data and drafting of manuscript: K.K. and B.D. Analysis and interpretation of data: K.K. B.D., S.S., and A.A. Critical revision: K.K., B.D., S.S., and S.K.M. Overall guidance: S.KM

\section{Conflict of interest}

The authors declare that they have no conflict of interest.

Supplementary Information accompanies this paper at https://doi.org/ 10.1038/s41419-017-0224-z.

Received: 13 September 2017 Revised: 11 December 2017 Accepted: 13 December 2017

Published online: 02 February 2018

\section{References}

1. Siegel, R. L., Miller, K. D. \& Jemal, A. Cancer Statistics, 2017. Ca. Cancer J. Clin. 67, 7-30 (2017).

2. Goel, S., Tripathy, J. P., Singh, R. J. \& Lal, P. Smoking trends among women in India: analysis of nationally representative surveys (1993-2009). South Asian J. Cancer 3, 200-202 (2014).

3. Palmer, J. R. \& Rosenberg, L. Cigarette smoking and the risk of breast cancer. Epidemiol. Rev. 15, 145-156 (1993).

4. Terry, P. D. \& Rohan, T. E. Cigarette smoking and the risk of breast cancer in women: a review of the literature. Cancer Epidemiol. Biomark. Prev. 11(Pt 1), 953-971 (2002).

5. Luo, J. et al. Association of active and passive smoking with risk of breast cancer among postmenopausal women: a prospective cohort study. BMJ $\mathbf{3 4 2}$, d1016 (2011)

6. Talhout, R. et al. Hazardous compounds in tobacco smoke. Int. J. Environ. Res. Public. Health 8, 613-628 (2011).

7. Pomerleau, O. F. Nicotine and the central nervous system: biobehavioral effects of cigarette smoking. Am. J. Med. 93, 2S-7S (1992).

8. Conway, K. et al. Prevalence and spectrum of p53 mutations associated with smoking in breast cancer. Cancer Res. 62, 1987-1995 (2002).

9. Li, D., Zhang, W., Sahin, A. A. \& Hittelman, W. N. DNA adducts in normal tissue adjacent to breast cancer: a review. Cancer Detect. Prev. 23, 454-462 (1999).

10. Rundle, A. et al. The relationship between genetic damage from polycyclic aromatic hydrocarbons in breast tissue and breast cancer. Carcinogenesis 21, 1281-1289 (2000)

11. Morabia, A., Bernstein, M., Heritier, S. \& Khatchatrian, N. Relation of breast cancer with passive and active exposure to tobacco smoke. Am. J. Epidemiol. 143, 918-928 (1996).

12. Reynolds, P. et al. Active smoking, household passive smoking, and breast cancer: evidence from the California Teachers Study. J. Natl. Cancer Inst. 96 , 29-37 (2004).
13. Govind, A. P., Vezina, P. \& Green, W. N. Nicotine-induced upregulation of nicotinic receptors: underlying mechanisms and relevance to nicotine addiction. Biochem. Pharmacol. 78, 756-765 (2009).

14. Lee, C. H. et al. Overexpression and activation of the alpha9-nicotinic receptor during tumorigenesis in human breast epithelial cells. J. Natl. Cancer Inst. 102, 1322-1335 (2010).

15. Wu, C. H., Lee, C. H. \& Ho, Y. S. Nicotinic acetylcholine receptor-based blockade: applications of molecular targets for cancer therapy. Clin. Cancer Res. 17 3533-3541 (2011)

16. Dasgupta, P. et al. Nicotine induces cell proliferation, invasion and epithelialmesenchymal transition in a variety of human cancer cell lines. Int. J. Cancer 124, 36-45 (2009)

17. Tran, T. Q., Lowman, X. H. \& Kong, M. Molecular pathways: metabolic control of histone methylation and gene expression in cancer. Clin. Cancer Res. 23, 4004-4009 (2017)

18. Behrens, C. et al. EZH2 protein expression associates with the early pathogenesis, tumor progression, and prognosis of non-small cell lung carcinoma. Clin. Cancer Res. 19, 6556-6565 (2013).

19. Chase, A. \& Cross, N. C. Aberrations of EZH2 in cancer. Clin. Cancer Res. 17 2613-2618 (2011)

20. Kleer, C. G. et al. EZH2 is a marker of aggressive breast cancer and promotes neoplastic transformation of breast epithelial cells. Proc. Natl. Acad. Sci. USA 100, 11606-11611 (2003)

21. Tan, J. et al. Pharmacologic disruption of Polycomb-repressive complex 2mediated gene repression selectively induces apoptosis in cancer cells. Genes Dev. 21, 1050-1063 (2007)

22. Perrot-Applanat, M. \& Di Benedetto, M. Autocrine functions of VEGF in breas tumor cells: adhesion, survival, migration and invasion. Cell Adhes. Migr. 6, 547-553 (2012)

23. Goel, H. L. \& Mercurio, A. M. VEGF targets the tumour cell. Nat. Rev. Cancer $\mathbf{1 3}$ 871-882 (2013).

24. Dong, W. et al. Matrix metalloproteinase 2 promotes cell growth and invasion in colorectal cancer. Acta Biochim. Biophys. Sin. (Shanghai). 43, 840-848 (2011).

25. Conklin, B. S., Zhao, W., Zhong, D. S. \& Chen, C. Nicotine and cotinine upregulate vascular endothelial growth factor expression in endothelial cells. Am. J. Pathol. 160, 413-418 (2002).

26. Zong, Y., Zhang, S. T. \& Zhu, S. T. Nicotine enhances migration and invasion of human esophageal squamous carcinoma cells which is inhibited by nimesulide. World J. Gastroenterol. 15, 2500-2505 (2009).

27. Paul-Samojedny, M. et al. Expression of cell survival/death genes: BCl-2 and Bax at the rate of colon cancer prognosis. Biochim. Biophys. Acta 1741, 25-29 (2005).

28. Dasgupta, P. et al. Nicotine inhibits apoptosis induced by chemotherapeutic drugs by up-regulating XIAP and survivin. Proc. Natl. Acad. Sci. USA 103 6332-6337 (2006)

29. Lu, J. Y. D. et al. The neuroprotective effect of nicotine in Parkinson's disease models is associated with inhibiting PARP-1 and caspase-3 cleavage. Peer J $\mathbf{5}$, e3933 (2017).

30. Jin, Q, Menter, D. G, Mao, L, Hong, W. K. \& Lee, H. Y. Survivin expression in normal human bronchial epithelial cells: an early and critical step in tumorigenesis induced by tobacco exposure. Carcinogenesis 29, 1614-1622 (2008).

31. Dinicola, S. et al. Nicotine increases survival in human colon cancer cells treated with chemotherapeutic drugs. Toxicol. In Vitro 27, 2256-2263 (2013).

32. Neri, F. et al. Myc regulates the transcription of the PRC2 gene to control the expression of developmental genes in embryonic stem cells. Mol. Cell Biol. 32, 840-851 (2012)

33. Shaul, Y. D. et al. MERAV: a tool for comparing gene expression across human tissues and cell types. Nucleic Acids Res. 44, D560-566 (2016).

34. Yamaguchi, H. \& Hung, M. C. Regulation and role of EZH2 in cancer. Cancer Res. Treat. 46, 209-222 (2014)

35. Sander, S. et al. MYC stimulates EZH2 expression by repression of its negative regulator miR-26a. Blood 112, 4202-4212 (2008).

36. Wang, L. et al. c-Myc-mediated epigenetic silencing of MicroRNA-101 contributes to dysregulation of multiple pathways in hepatocellular carcinoma. Hepatology 59, 1850-1863 (2014)

37. Varambally, S. et al. Genomic loss of microRNA-101 leads to overexpression of histone methyltransferase EZH2 in cancer. Science 322, 1695-1699 (2008).

38. Dang, $X$. et al. MicroRNA-26a regulates tumorigenic properties of EZH2 in human lung carcinoma cells. Cancer Genet. 205, 113-123 (2012). 
39. Yu, M. A. et al. Nicotine promotes acquisition of stem cell and epithelial-tomesenchymal properties in head and neck squamous cell carcinoma. PLoS ONE 7, e51967 (2012).

40. Bavarva, J. H., Tae, H., Settlage, R. E. \& Garner, H. R. Characterizing the genetic basis for nicotine induced cancer development: a transcriptome sequencing study. PLOS ONE 8, e67252 (2013).

41. Hao, J. et al. Nicotinic receptor beta2 determines NK cell-dependent metastasis in a murine model of metastatic lung cancer. PLOS ONE 8, e57495 (2013).

42. Hogg, N. Nicotine has suppressive effects on dendritic cell function. Immunology 109, 329-330 (2003).

43. Nouri-Shirazi, M. \& Guinet, E. Evidence for the immunosuppressive role of nicotine on human dendritic cell functions. Immunology 109, 365-373 (2003).

44. McAllister-Sistilli, C. G. et al. The effects of nicotine on the immune system. Psychoneuroendocrinology 23, 175-187 (1998).

45. Fleming, J. M., Miller, T. C., Meyer, M. J., Ginsburg, E. \& Vonderhaar, B. K. Local regulation of human breast xenograft models. J. Cell. Physiol. 224, 795-806 (2010).

46. Price, J. E., Polyzos, A., Zhang, R. D. \& Daniels, L. M. Tumorigenicity and metastasis of human breast carcinoma cell lines in nude mice. Cancer Res. $\mathbf{5 0}$ 717-721 (1990).

47. Clarke, R. Human breast cancer cell line xenografts as models of breast cancer The immunobiologies of recipient mice and the characteristics of several tumorigenic cell lines. Breast Cancer Res. Treat. 39, 69-86 (1996).
48. Carreno, B. M. et al. Immunodeficient mouse strains display marked variability in growth of human melanoma lung metastases. Clin. Cancer Res. 15, 3277-3286 (2009).

49. Miranda, T. B. et al. DZNep is a global histone methylation inhibitor that reactivates developmental genes not silenced by DNA methylation. Mol. Cancer Ther. 8, 1579-1588 (2009).

50. Faustino-Rocha, A. et al. Estimation of rat mammary tumor volume using caliper and ultrasonography measurements. Lab. Anim. 42, 217-224 (2013).

51. Watson, A. S. \& Soilleux, E. J. Detection of p62 on paraffin sections by immunohistochemistry. Cold Spring Harb. Protoc. 2015, 756-760 (2015).

52. McDonald, J. W. \& Pilgram, T. K. Nuclear expression of p53, p21 and cyclin D1 is increased in bronchioloalveolar carcinoma. Histopathology 34, 439-446 (1999).

53. van Meerloo, J., Kaspers, G. J. \& Cloos, J. Cell sensitivity assays: the MTT assay Methods Mol. Biol. 731, 237-245 (2011).

54. Justus C. R., Leffler N., Ruiz-Echevarria M. \& Yang L. V. In vitro cell migration and invasion assays. J. Vis. Exp. 88, 51046 (2014).

55. Rio, D. C., Ares, M. Jr., Hannon, G. J. \& Nilsen, T. W. Purification of RNA using TRIzol (TRI reagent). Cold Spring Harb. Protoc. 2010, pdbprot5439 (2010).

56. De Haan, G. \& Gerrits, A. Epigenetic control of hematopoietic stem cell aging the case of Ezh2. Ann. NY Acad. Sci. 1106, 233-239 (2007).

57. Pereira, J. D et al. Ezh2, the histone methyltransferase of $P R C 2$, regulates the balance between self-renewal and differentiation in the cerebral cortex. Proc. Natl. Acad. Sci. USA 107, 15957-15962 (2010). 